\title{
Tolerância da cultura do tomate à salinidade do solo em ambiente protegido ${ }^{1}$
}

\author{
Pedro R. F. Medeiros ${ }^{2}$, Sergio N. D uarte ${ }^{3}$, Cláudio A. Uyeda ${ }^{4}$, \\ Ênio F. F. Silva ${ }^{5} \&$ José F. de Medeiros ${ }^{6}$
}

\begin{abstract}
RESU M O
Com o intuito de contribuir com os dados disponíveis na literatura sobre tolerância das culturas à salinidade do solo oriunda de sais fertilizantes, o presente trabalho tem, como objetivo, determinar a tolerância da cultura do tomate a salinidade do solo, a partir de dois manejos de fertirrigação e seis níveis iniciais de salinidade $\left(1,0 ; 2,0 ; 3,0 ; 4,0 ; 5,0\right.$ e $\left.6,0 \mathrm{dS} \mathrm{m}^{-1}\right)$ a partir das variáveis produção e componentes de produção, em ambiente protegido com solo franco-argiloso. 0 experimento foi realizado no Departamento de Engenharia de Biossistemas da ESALQ/USP, Piracicaba,SP. 0 delineamento estatístico foi aleatorizado em blocos, com 4 repetições, concluindo que a produção e os componentes de produção da cultura do tomate foram afetados estatisticamente, tanto pelo tipo de manejo de fertirrigação quanto pelos níveis de salinidade do solo, com redução na tolerância da cultura do tomate, passando a ser classificada sensível à salinidade do solo.
\end{abstract}

Palavras chave: irrigação, fertilizantes, produção relativa

\section{Tolerance of tomato crop to salinity of soil in protected ambient}

\begin{abstract}
In order to contribute to data available in the literature on tolerance of crops to soil salinity derived from fertilizer salts, the present study aims to determine the tolerance of tomato crop to the soil salinity, based on two managements of fertirrigation and six initial levels of salinity $\left(1.0 ; 2.0 ; 3.0 ; 4.0 ; 5.0\right.$ and $\left.6.0 \mathrm{dS} \mathrm{m}^{-1}\right)$ from the variables production and components of production, in a protected environment with sandyclay soil. The experiment was conducted at the Department of Biosystems Engineering of ESALQ/USP, Piracicaba/SP. The experimental design was in randomized blocks, with 4 repetitions. Concluding that the production and the components of production of tomato crop were significantly affected both by the type of management of fertirrigation and by the levels of soil salinity, with a reduction in the tolerance of tomato crop, being classified as sensitive to soil salinity.
\end{abstract}

Key words: Irrigation, fertilizers, relative production

\footnotetext{
${ }^{1}$ Parte da Tese de Doutorado do primeiro autor, apresentada à ESALQ/U SP, Piracicaba, SP

2 D outorando em Irrigação e Drenagem, ESALQ/U SP. Av. Pádua Dias, 11. CEP 13418-900. Piracicaba,SP. Fone: (19) 3447-8561. E-mail: prfmede@yahoo.com.br

3 ESALQ/U SP. Fone: (19) 3447-8543. E-mail: snduarte@esalq.usp.br

${ }^{4}$ IFPB, Campus Picuí. Acesso Rod. PB 151, s/n, Bairro Cenecista, CEP 58187-000, Picuí, PB. Fone: (83) 3371-2555. E-mail: cauyeda@yahoo.com.br

5 U FRPE, Rua D. Manoel de Medeiros s/n, CEP 52171-900 Recife, PE. Fone (81) 3320-6261. E-mail: enio.silva@dtr.ufrpe.br

${ }^{6}$ U FERSA, BR 110, C.P. 137, CEP 59625-900, Mossoró-RN. Fone (84) 33151740. E-mail: jfmedeir@ufersa.edu.br
} 


\section{INTRODUÇÃO}

A salinidade do solo, oriunda do uso indevido de sais fertilizantes altamente solúveis e facilmente transportados na água, é percussora de danos bem mais severos ao solo e às plantas, principalmente se combinadas os fatores, tipo, concentração dos sais e suas interações na relação água-soloplanta. Esta salinidade pode ser oriunda do manejo inadequado da fertirrigação, causa atualmente frequente em muitas regiões, principalmente em ambiente protegido.

O acúmulo de sais no solo em cultivos protegidos é bastante comum devido, principalmente, às altas doses de fertilizantes aplicados e a falta de lixiviação dos sais acumulados após um cultivo. A aplicação de doses elevadas de $\mathrm{N}$ potencializa os efeitos da salinidade, reduzindo a tolerância da cultura do tomate (Blanco \& Folegatti, 2008).

Parte desses sais fertilizantes adicionados ao solo junto com a água de irrigação (técnica da fertirrigação) é consumida e o restante se deposita nos colóides do solo com potencial para aumentar a concentração, sobretudo depois de sucessivas aplicações de fertirrigação, podendo chegar a ponto de reduzir o desenvolvimento vegetativo das culturas e posteriormente, o rendimento.

Este tipo de salinidade deve ter um manejo de forma diferenciada, a fim de se evitar o desperdício de fertilizantes a partir do monitoramento dos íons e da condutividade elétrica da solução do solo, buscando-se sempre um ponto ótimo de tolerância para as plantas, com o uso de equipamentos que determinem, com precisão e rapidez, a melhor eficiência da técnica. Solos afetados por sais podem ser explorados economicamente desde que se adote um manejo adequado do sistema solo-água-planta (Rhoades et al.,2000).

Nem todas as culturas respondem igualmente à salinidade; algumas produzem rendimentos aceitáveis a altos níveis de salinidade e outras são sensíveis a níveis relativamente baixos, cuja diferença se deve à melhor capacidade osmótica que algumas culturas têm o que permite absorver, mesmo em condições de salinidade, maior quantidade de água (Ayers \& Westcot, 1999). Segundo Medeiros et al. (2008), estudando o manejo da frequência de irrigação com água salina, a cultivar de melão Trusty é mais tolerante à salinidade que a Orange flesh.

A salinidade máxima do extrato de saturação do solo tolerada pelo tomateiro, é de 2,5 dS m m $^{-1}$ (Maas \& Hoffman, 1977) classificada como moderadamente sensível, embora possa existir resposta diferenciada à salinidade entre as diferentes cultivares (Alian et al., 2000). Sob salinidade elevada, a redução na produtividade é resultado do menor número de frutos por planta.

Em geral, a salinidade promove um desbalanço nutricional nas plantas em virtude da competição entre os sais e os nutrientes no processo de absorção (Demiral, 2005) e poucos estudos têm sido conduzidos para avaliar a resposta do tomateiro à adubação sob condições de salinidade.

O nível máximo de salinidade média da zona radicular que pode ser tolerado pelas plantas sem afetar negativamente seu desenvolvimento é a salinidade limiar (SL) (Maas \& Hoffman, 1977). A esta salinidade expressa em termos da condutividade elétrica do extrato de saturação do solo, o rendimento potencial das culturas é $100 \%$.

Tendo em vista a falta de resultados científicos envolvendo a tolerância das culturas sobre a salinização ocasionada pelo excesso de fertilizantes propõe-se, neste trabalho, avaliar a cultura do tomate em relação à tolerância a salinidade do solo, a partir de dois manejos de fertirrigação e seis níveis iniciais de salinidade $\left(1,0 ; 2,0 ; 3,0 ; 4,0 ; 5,0\right.$ e $\left.6,0 \mathrm{dS} \mathrm{m}^{-1}\right)$, sendo monitorados por extratores de cápsulas porosas, sobre as variáveis produção e componentes de produção, em ambiente protegido com solo franco-argiloso.

\section{Material e MÉTODOS}

O experimento foi conduzido em ambiente protegido na área experimental do Departamento de Engenharia de Biossistemas da ESALQ/USP/SP, com a cultura do tomate com a cultivar Débora Plus, por ser uma cultivar bastante plantada no estado de São Paulo.

A irrigação utilizada foi a localizada com o método de gotejamento, os emissores com vazão nominal de $4 \mathrm{~L} \mathrm{~h}^{-1}$. Para auxiliar as irrigações, o método de manejo por tensiometria foi utilizado adotando-se o seguinte manejo, as irrigações foram realizadas quando a tensão na cápsula cerâmica dos tensiômetros atingia $20 \mathrm{kPa}$ e somente durante o tempo necessário para que a umidade do solo retornasse à capacidade de campo $(5 \mathrm{kPa})$.

O solo utilizado foi o franco-argiloso (Tabela 1) acondicionado em vasos de $25 \mathrm{~L}$ nos quais realizou-se o plantio das plantas. O sistema de poda foi o de haste única, com desbastes semanais dos brotos nas axilas das folhas. O sistema de tutoramento foi na vertical, com o auxílio de fitilhos e arames lisos. Os tratamentos fitossanitários eram realizados semanalmente, com o objetivo de manter o stand sempre livre de pragas e doenças a fim de evidenciar cada vez mais o efeito da salinidade do solo causada por sais fertilizantes.

Tabela 1. Características químicas e físicas do solo

\begin{tabular}{|c|c|c|c|c|c|c|}
\hline \multirow{2}{*}{$\begin{array}{c}\mathrm{pH} \\
\mathrm{CaCl}^{2}\end{array}$} & $\bar{K}$ & $\mathrm{Ca}$ & $\mathrm{Mg}$ & $\mathrm{H}+\mathrm{Al}$ & S.B. & \multirow{2}{*}{ СТC } \\
\hline & \multicolumn{5}{|c|}{$\mathrm{mmol}_{\mathrm{c}} \mathrm{dm}^{-3}$} & \\
\hline \multicolumn{7}{|c|}{ Características químicas } \\
\hline 5,1 & 6,9 & 29 & 11 & 28 & 46,9 & 74,9 \\
\hline CC & PMP & \multirow{2}{*}{$\begin{array}{c}\mathrm{Dg} \\
\mathrm{g} \mathrm{cm}^{-3}\end{array}$} & \multicolumn{3}{|c|}{ Frações - \% } & \multirow{2}{*}{ Textura } \\
\hline & & & Argila & Silte & Areia & \\
\hline \multicolumn{3}{|c|}{ Características físicas } & & & & \\
\hline 0,41 & 0,05 & 1,25 & 41,23 & 13,68 & 45,09 & Franco Argilosa \\
\hline
\end{tabular}

Os tratamentos foram os seguintes: dois tipos de manejo de fertirrigação (M1 e M2) interagindo com seis níveis iniciais de salinidade do solo $\left(1,0 ; 2,0 ; 3,0 ; 4,0 ; 5,0\right.$ e $\left.6,0 \mathrm{dS} \mathrm{m}^{-1}\right)$ a partir de sais fertilizantes.

O manejo M1, que é o manejo tradicional de fertirrigação, obedeceu à recomendação de adubação proposta por Alvarenga (2004) para acultura do tomate; já o manejo M2, teve como objetivo principal controlar o nível inicial de salinidade do solo através de medições da condutividade 
elétrica da solução do solo relacionadas com concentrações de sais fertilizantes a partir de curvas artificiais de salinização induzida. A condutividade elétrica da solução do solo obtida com extratores de cápsula porosa, era corrigida pelo método padrão da pasta saturada (Eq. 1), para determinação da salinidade do solo.

$$
\mathrm{C}_{\text {estimada }} \mathrm{cp}=\mathrm{Ccp} . \mathrm{Ucp} / \mathrm{Us}
$$

em que:

$\mathrm{C}_{\text {estimada }} \mathrm{cp}$ - condutividade elétrica ou concentração de íons no extrato de saturação, estimada a partir dos valores medidos na solução do solo obtida com extrator de cápsula, dS m

Ccp - condutividade elétrica ou concentração de íons na solução do solo, obtida com extrator de cápsula porosa, $\mathrm{dS} \mathrm{m}^{-1}$

Ucp - umidade do solo no momento da retirada da solução com o extrator de cápsula porosa, $\mathrm{g} \mathrm{g}^{-1}$

Us - umidade do solo na pasta saturada, $\mathrm{g} \mathrm{g}^{-1}$

Os seis níveis de salinidade do solo foram aplicados antes do transplantio independente do manejo de fertirrigação adotado, de forma a simular solos salinos em seis diferentes níveis provocados por excesso de sais fertilizantes em ambiente protegido. A salinização inicial do solo foi por meio da aplicação de solução salina a partir da mistura dos sais fertilizantes (Eloi et al., 2007; Medeiros, 2007).

As parcelas foram distribuídas (sorteadas) em quatro blocos, sendo cada bloco considerado uma repetição. O delineamento estatístico adotado foi o aleatorizado em blocos completos com quatro repetições, ficando os fatores estudados arranjados no esquema fatorial $6 \times 2$, totalizando 48 unidades experimentais. As variáveis respostas analisadas foram produção total (PRODT) e comercial (PRODC) ambas em $\mathrm{Mg} \mathrm{ha}^{-1}$, número de frutos comerciais (NUMC) e totais (NUMT).

A partir dos dados de produção total calculou-se o rendimento relativa da cultura do tomate com o propósito de determinar a salinidade limiar (SL), utilizando-se a Eq. 2 (Maas \& Hoffman (1977).

$$
\mathrm{Y}=100-\text { b. }(\text { CEes }-\mathrm{SL})
$$

em que:

$\mathrm{Y}$ - rendimento potencial, \%

CEes - salinidade do extrato de saturação, $\mathrm{dS} \mathrm{m}^{-1}$

$\mathrm{SL}$ - salinidade limiar da cultura, $\mathrm{dS} \mathrm{m}^{-1}$

b - diminuição do rendimento por aumento unitário de salinidade acima do valor de SL.

\section{RESULTADOS E DISCUSSÃO}

A produção de tomate é considerada atividade de alto risco, devido principalmente à grande variedade de ambientes e sistemas nos quais é cultivado. A salinidade do solo, o manejo de fertirrigação e a interação entre ambos, afetaram as variáveis produção total, produção comercial e número de frutos comerciais a nível de $1 \%$ de probabilidade com efeito linear (Tabelas 2). Segundo Medeiros et al. (2008), a salinidade da água de irrigação interferiu significativamente sobre as produtividades comerciais e totais, os números de frutos comerciais e totais da cultura do melão.

Tabela 2. Resumo da análise de variância para a variável produção total (PRODT) e comercial (PRODC) ambas em Mg ha-1, número de frutos comerciais (NUMC) e totais (NU MT), submetidos a diferentes níveis de salinidade do solo e tipos de manejo de fertirrigação

\begin{tabular}{lcccc}
\hline \multirow{2}{*}{ Fontes de variação } & \multicolumn{4}{c}{ Estatística F } \\
\cline { 2 - 5 } & PRODT & PRODC & NUMT & NUMC \\
- Salinidade (S) & $0,14^{* *}$ & $0,34^{* *}$ & $1,08^{\text {ns }}$ & $20,24^{* *}$ \\
Linear & $0,64^{* *}$ & $1,61^{* *}$ & $1,30^{\text {ns }}$ & $95,69^{* *}$ \\
Quadrática & $0,07^{\text {ns }}$ & $3,09^{\text {ns }}$ & $0,31^{\text {ns }}$ & $0,02^{\text {ns }}$ \\
- Manejo (M) & $0,17^{* *}$ & $0,78^{* *}$ & $0,20^{\text {ns }}$ & $47,40^{* *}$ \\
(S) x (M) & $0,09^{* *}$ & $0,20^{* *}$ & $1,81^{\text {ns }}$ & $10,94^{* *}$ \\
CV (\%) & 22,49 & 35,73 & 23,76 & 40,63 \\
\hline Manejos & & & & \\
M1 & $8,4 b$ & $2,2 b$ & $23,27 a$ & $3,35 b$ \\
M2 & $11,0 a$ & $5,9 a$ & $22,56 a$ & $7,89 a$ \\
\hline
\end{tabular}

* e** significativo a $5 \%$ e a $1 \%$ de probabilidade, respectivamente. ns - não significativo Médias seguidas da mesma letra não diferem entre si pelo teste de Tukey ao nível de 0,05 de probabilidade

M1 - manejo tradicional de fertirrigação

M2 - manejo mediante controle da salinidade inicial do solo

Em relação à quantificação da precisão do experimento, observa-se uma variação relativa $(\mathrm{CV})$ classificada média, para produção total (PRODT), produção comercial (PRODC), número de frutos totais (NUMT) e muito alta para número de frutos comerciais (NUMC) (Lima et. al., 2004).

Observam-se, na Figura 1, as análises de regressão para as variáveis produção total, produção comercial e número de frutos comerciais, todas lineares, decrescendo ao percentual de 0,$24 ; 2,72 ; 0,37 ; 2,73 ; 0,50$ e $3,26 \%$ com o aumento de uma unidade da salinidade do solo, para produção total no manejo de fertirrigação M1 (Figura 1A), produção total no manejo de fertirrigação M2 (Figura 1B), produção comercial no manejo de fertirrigação M1 (Figura 1C), produção comercial no manejo de fertirrigação M2 (Figura 1D), número de frutos comerciais no manejo de fertirrigação M1 (Figura 1E) e número de frutos comerciais no manejo de fertirrigação M2 (Figura 1F), respectivamente.

Araújo et al. (2009) afirmam que o diâmetro, peso médio eo número de frutos total e comercial do pimentão, são influenciados de forma linear e positiva pelas doses crescentes de nitrogênio.

Pela tendência, os níveis mais baixos de salinidade do solo foram os mais produtivos para todas as análises significativas, resultado também encontrado quando se utilizaram a mesma metodologia e cultura com solo franco-arenoso (Eloi et al., 2007).

$\mathrm{Na}$ Tabela 3 se encontram as médias referentes à interação entre os fatores salinidade do solo e manejo de fertirrigação, evidenciando diferenças significativas tanto entre os níveis de salinidade do solo quanto entre tipo de manejo de fertirrigação para as variáveis PRODT, PRODC e NUMC. O manejo de fertirrigação M2 obteve as maiores médias principalmente quando associado com os níveis mais baixos de salinidade e o nível de salinidade S6 obteve as menores médias no geral. 
A.

B.
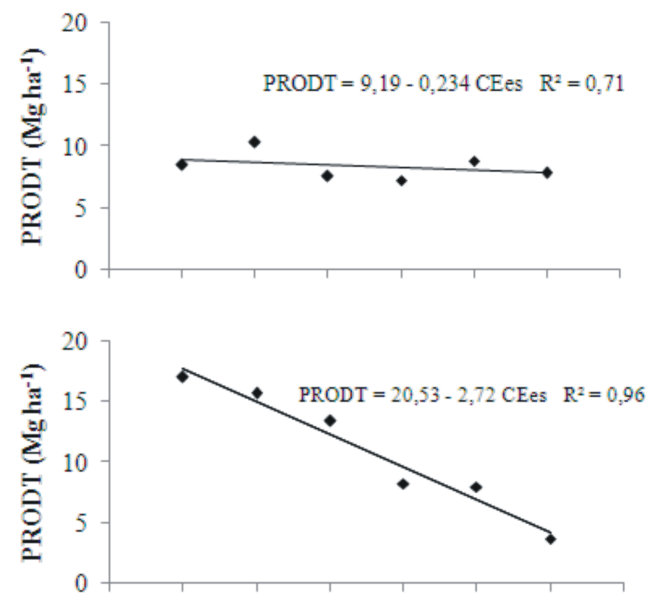

C.



D.

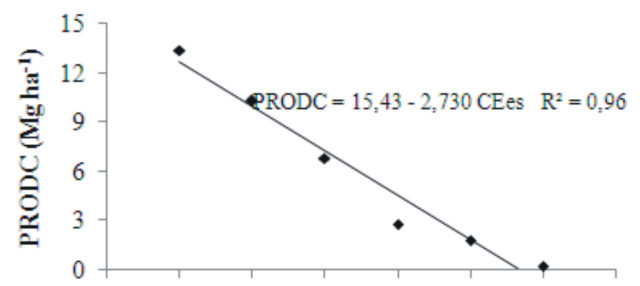

E

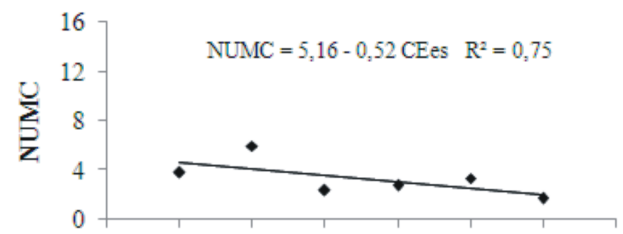

F.

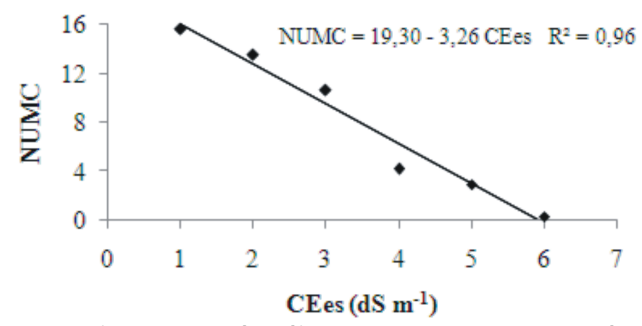

Figura 1. Diagrama de dispersão e equação de ajuste para produção total (PRODT), produção comercial (PRO DC) e número de frutos comerciais (N U MC) em função da salinidade do solo e do tipo de manejo de fertirrigação $M 1$ ( $A, C$ e E) e M 2 (B, D e F)

Observa-se, na Figura 2, o gráfico de tolerância da cultura do tomate. A Salinidade Limiar foi 1,278 $\left(\mathrm{dS} \mathrm{m}^{-1}\right)$, bem abaixo da encontrada na literatura evidenciando o efeito agressivo da salinidade do solo por sais fertilizantes em solo franco-argiloso, e um decréscimo relativo da produtividade de $15,96 \%$ para cada aumento de uma unidade da salinidade do solo. Segundo
Tabela 3. Valores médios* da variável produção total (PRODT (Mg ha-1)), produção comercial (PRODC $\left(\mathrm{M} \mathrm{g} \mathrm{ha}^{-1}\right)$ ) e número de frutos comerciais (NU MC) entre tipo de manejo de fertirrigação e os níveis de salinidade do solo

\begin{tabular}{crrrrrrrr}
\hline Níveis de & \multicolumn{2}{c}{ PRODT } & & \multicolumn{2}{c}{ PRODC } & & \multicolumn{2}{c}{ NUMC } \\
\cline { 2 - 3 } salinidade $^{*}$ & M1 & M2 & & M1 & M2 & & M1 & M2 \\
S1 & $8,5 \mathrm{Ba}$ & $17,1 \mathrm{Aa}$ & & $2,6 \mathrm{Ba}$ & $13,4 \mathrm{Aa}$ & & $3,87 \mathrm{Bb}$ & $15,62 \mathrm{Aa}$ \\
S2 & $10,3 \mathrm{Ba}$ & $15,7 \mathrm{Aa}$ & & $4,0 \mathrm{Ba}$ & $10,3 \mathrm{Ab}$ & & $6,00 \mathrm{Ba}$ & $13,50 \mathrm{Aa}$ \\
S3 & $7,6 \mathrm{Ba}$ & $13,4 \mathrm{Aa}$ & & $1,5 \mathrm{Bb}$ & $6,8 \mathrm{AC}$ & & $2,37 \mathrm{Bb}$ & $10,62 \mathrm{aa}$ \\
S4 & $7,2 \mathrm{Aa}$ & $8,2 \mathrm{Ab}$ & & $1,8 \mathrm{Ab}$ & $2,8 \mathrm{Ad}$ & & $2,75 \mathrm{Ab}$ & $4,25 \mathrm{Ab}$ \\
S5 & $8,8 \mathrm{Aa}$ & $8,0 \mathrm{Ab}$ & & $2,3 \mathrm{Ab}$ & $1,8 \mathrm{Ad}$ & & $3,37 \mathrm{Ab}$ & $3,00 \mathrm{Ab}$ \\
S6 & $7,8 \mathrm{Aa}$ & $3,7 \mathrm{BC}$ & & $1,0 \mathrm{Ab}$ & $0,2 \mathrm{Ae}$ & & $1,75 \mathrm{Ab}$ & $0,37 \mathrm{Ab}$ \\
\hline
\end{tabular}

* Médias seguidas da mesma letra maiúsculas nas linhas e minúsculas nas colunas não diferem entre si pelo teste de Tukey a nível de 0,05 de probabilidade

** S1 a S6 - referem-se a CE $\mathrm{E}_{\text {es }}$ do solo 1, 2, 3, 4, 5 e $6 \mathrm{dS} \mathrm{m}^{-1}$

Rhoades et al. (1992), para o tomateiro o valor de salinidade limiar, expresso em termos de condutividade elétrica do extrato de saturação, é de $2,5 \mathrm{dS} \mathrm{m}^{-1}$ com decréscimo relativo de produtividade por unidade de CEes para a cultura de $9,0 \%$.

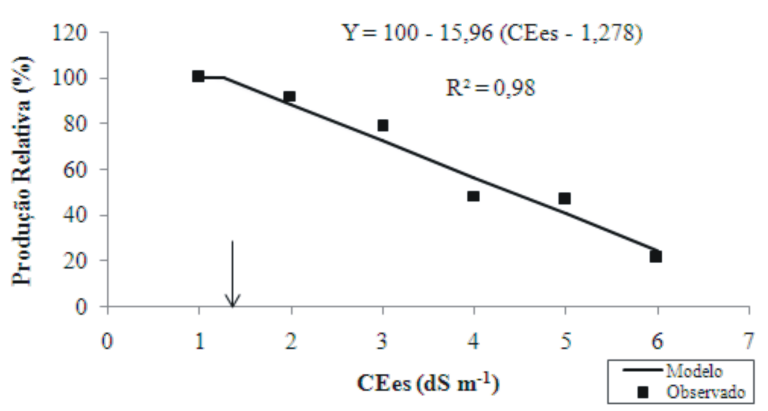

Figura 2. Rendimento relativo para as médias da produtividade ( $\mathrm{M} \mathrm{g} \mathrm{ha}^{-1}$ ) em função as salinidade do solo

Em geral, a salinidade do solo, tanto causada pela irrigação com água salina como pela combinação dos fatores água e solo, pode resultar em redução da produção, mudanças de cor e de aparência e alterações na qualidade do produto colhido (Rhoades et al., 2000).

Eloi et al. (2007), utilizando a mesma cultura e metodologia com solo franco-arenoso, encontraram o valor de Salinidade Limiar de 3,03 $\left(\mathrm{dS} \mathrm{m}^{-1}\right)$, com decréscimo relativo da produtividade de $10,95 \%$ para cada aumento de uma unidade da salinidade do solo, causada por sais fertilizantes.

Medeiros (2007), utilizando a mesma metodologia com a cultura do pepino e solo franco arenoso, encontrou valores de Salinidade Limiar de 4,08 ( $\left.\mathrm{dS} \mathrm{m}^{-1}\right)$, com decréscimo relativo da produtividade de $19,33 \%$ para cada aumento de uma unidade da salinidade do solo, causada por sais fertilizantes.

Medeiros et al. (2009), estudando a tolerância da cultura do pepino à salinidade em ambiente protegido, verificaram que a cultura apresentou valores satisfatórios nas suas variáveis respostas para o nível de salinidade do solo 3,5 $\mathrm{dS} \mathrm{m}^{-1}$.

A variedade de milho UFVM 100, mostrou-se sensível à salinidade do solo e a produção relativa decresceu 15,68\% com incrementos unitários da salinidade do solo acima de 1,70 $\mathrm{dS} \mathrm{m}^{-1}$ (salinidade limiar) (Ferreira et al., 2007).

$\mathrm{Na}$ Tabela 4 se encontra a taxa de frutos descartados em função dos níveis de salinidade do solo e do tipo de manejo de 
fertirrigação. Pela tendência, o nível de salinidade do solo mais produtivo foi o que teve menos perdas na produção, constatando-se o mesmo pra o manejo de fertirrigação M2.

Tabela 4. Valores médios (\%) representando a taxa de perda de produção, entre a produção total (PRODT) e comercial (PRODC) em função do manejo de fertirrigação e níveis de salinidade do solo

\begin{tabular}{ccccccc}
\hline Manejo de & \multicolumn{6}{c}{ Níveis de salinidade do solo } \\
\cline { 2 - 7 } fertirrigação & S1 & S2 & S3 & S4 & S5 & S6 \\
M1 & 69 & 61 & 81 & 76 & 74 & 87 \\
M2 & 21 & 34 & 49 & 66 & 78 & 94 \\
\hline M1 - manejo tradicional de fertirrigacão & & \multicolumn{5}{c}{}
\end{tabular}

M1 - manejo tradicional de fertirrigação

M2 - manejo mediante controle da salinidade inicial do solo

Elevadas taxas de perdas se encontram nos níveis mais elevados de salinidade do solo, devido, principalmente, ao problema fisiológico fundo preto dos frutos, seguido de deformações e frutos pequenos. O problema fisiológico é atribuído à deficiência de cálcio na planta devendo-se, em parte, à falta de calagem do solo antes da instalação do experimento como, também, a uma possível toxidez que originou a deficiência de cálcio, considerando-se que a aplicação de sais fertilizantes nesses níveis se deu de forma excessiva.

A salinidade aumenta a incidência de podridão apical (Martinez et al., 1987; Cuartero \& Muñoz, 1999), tornando os frutos inutilizáveis, tanto para consumo quanto para a indústria.

\section{ConclusõEs}

1. A produção e os componentes de produção (produção total e comercial, número de frutos totais e comerciais) da cultura do tomate, foram afetados estatisticamente, tanto pelo tipo de manejo de fertirrigação adotado quanto pelos níveis de salinidade do solo.

2. As maiores taxas de perdas de frutos ocorreram nos níveis mais elevados de salinidade do solo.

3. A partir dos resultados de rendimento relativo, observase diminuição na tolerância da cultura, sendo classificada sensível a salinidade do solo causada por sais fertilizantes, para o respectivo experimento.

\section{Agradecimentos}

Os autores agradecem ao Ministério da Ciência e Tecnologia (MCT), ao Conselho Nacional de Desenvolvimento Científico e Tecnológico (CNPq) e à Fundação de Amparo à Pesquisa do Estado de São Paulo (FAPESP), pelo apoio financeiro a esta pesquisa, através do Instituto Nacional de Ciência e Tecnologia em Engenharia da Irrigação (INCTEI).

\section{LITERATURA CITADA}

Alian, A.; Altman, A.; Heuer, B. Genotypic difference in salinity and water stress tolerance of fresh market tomato cultivars. Plant Science, v.152, p.59-65, 2000.

Alvarenga, M. A. R. Tomate: Produção em campo, em casa-devegetação e em hidroponia. Lavras: UFLA, 2004. 400p.

Araújo, J. S.; Andrade, A. P.; Ramalho, C. I.; Azevedo, C. A. V. Cultivo do pimentão em condições protegidas sob diferentes doses de nitrogênio via fertirrigação. Revista Brasileira de Engenharia Agrícola e Ambiental, v.13, p.559$565,2009$.

Ayers, R. S.; Westcot, D. W. A qualidade de água na agricultura. 2.ed. Campina Grande: UFPB, 1999. 153p. FAO. Estudos Irrigação e Drenagem, 29

Blanco, F. F.; Folegatti, M. V. Doses de N e K no tomateiro sob estresse salino: III. Produção e qualidade de frutos. Revista Brasileira de Engenharia Agrícola e Ambiental, v.12, p.122127, 2008.

Cuartero, J.; Muñoz, R. F. Tomato and salinity. Scientia Horticulturae, v.78, p.83-125, 1999.

Demiral, M. A. Comparative response of two olive (Olea europaea L.) cultivars to salinity. Turkish Journal of Agriculture and Forestry, v.29, p.267-274, 2005.

Eloi, W. M.; Duarte, S. N.; Soares, T. M. Níveis de salinidade e manejo da fertirrigação sobre características do tomateiro cultivado em ambiente protegido. Revista Brasileira de Ciências Agrárias, v.2, p.83-89, 2007.

Ferreira, P.A.; Garcia, G O.; Neves, J. C. L.; Miranda, G V.; Santos, D. B. Produção relativa do milho e teores folheares de nitrogênio, fósforo, enxofre e cloro em função da salinidade do solo. Revista Ciência Agronômica, v.38, p.7-16, 2007.

Maas, E. V.; Hoffman, G. J. Crop salt tolerance - Current Assessment. Journal of Irrigation and Drainage Division, v.103, p.115-134, 1977.

Martinez, V.; Cerda, A.; Fernandez, F. G. Salt tolerance of four tomato hybrids. Plant and Soil, v.97, p.233-242, 1987.

Medeiros, J. F.; Dias, N. S.; Barros, A. D. Manejo da irrigação e tolerância do meloeiro a salinidade da água de irrigação. Revista Brasileira de Ciências Agrárias, v.3, p.242-247, 2008.

Medeiros, P. R. F. Manejo da fertirrigação em ambiente protegido visando o controle da salinidade para a cultura do pepino enxertado. Piracicaba: ESALQ/USP, 2007. 82p. Dissertação Mestrado

Medeiros, P. R. F.; Duarte, S. N.; Dias, C. T. S. Tolerância da cultura do pepino a salinidade em ambiente protegido. Revista Brasileira de Engenharia Agrícola e Ambiental, v.13, p.406-410, 2009.

Rhoades, J. D.; Kandiah, A.; Mashali, A. M. Uso de águas salinas para produção agrícola. Campina Grande: UFPB, 2000. 117p. Estudos da FAO, Irrigação e Drenagem, 48 